\title{
Ethical dilemmas in palliative care: a study in Taiwan
}

Tai-Yuan Chiu, Wen-Yu Hu, Shao-Yi Cheng and Ching-Yu Chen National Taiwan University Hospital and National Taiwan University, Taipei, Taiwan

\begin{abstract}
Objectives-To investigate the incidence and solution of ethical dilemmas in a palliative care unit. Design-Health care workers recorded daily all dilemmas in caring for each patient.

Setting-Palliative care unit of National Taiwan University Hospital in Taiwan.

Patients-Two hundred and forty-six consecutive patients with terminal cancer during 1997-8. Main measurement-Ethical dilemmas in the questionnaire were categorised as follows: telling the truth; place of care; therapeutic strategy; hydration and nutrition; blood transfusion; alternative treatment; terminal sedation; use of medication, and others. Results-The type and frequency of ethical dilemmas encountered were: place of care $(33.3 \%)$; truth-telling (32.1\%); hydration and nutrition (25.2\%); therapeutic strategy (24.8\%), and use of medication (19.1\%). Ethical problems relating to the place of care and to therapeutic strategy were unlikely to be solved with increased hospital stay and some ethical dilemmas remained unsolved even in the final week in hospital, including place of care $(23.2 \%)$, truth-telling $(17.1 \%)$ and therapeutic strategy (11.4\%). Problems of truth-telling occurred in nearly half (42.6\%) of patients over sixty-five-years-old. Conflicts about blood transfusion were experienced in all patients below 18-years-old, and the dilemmas concerning the place of care occurred most frequently with head and neck cancer patients $(43.8 \%)$.

Conclusions-The solution of ethical dilemmas required refocusing by medical professionals on the importance of continuing communication. Improved ethical training for professionals would contribute to solving the moral dilemmas of palliative care.

(Fournal of Medical Ethics 2000;26:353-357)

Keywords: Palliative care; terminal care; ethical dilemma
\end{abstract}

\section{Introduction}

Hospice and palliative care is well recognised as the ideal model of care for the terminally ill. As the numbers of terminal cancer patients in Taiwan have continued to increase in recent years, palliative care has been advocated as a moral responsibility. ${ }^{1-3}$ However, ethical issues are frequently encountered during the provision of hospice and palliative care. Bradshaw described how the spirit of hospice has been routinised, institutionalised and medicalised as the result of overemphasis on techniques and efficiency, thus deviating from its original goal. ${ }^{4}$
Kinzbrunner reported that the most frequently encountered ethical dilemmas in the US concerned predicting the survival of the terminally ill (as documented by the Medicare Hospice Benefit limitation of six months); truth-telling; hesitancy to use morphine for fear of causing respiratory failure; issues related to parenteral nutrition, and difficulty in meeting the needs of delirious patients. ${ }^{5}$ Finlay described difficult clinical decisions in hospice treatment in the UK such as the treatment of hypercalcaemia, uraemia, abnormal serum sugar level, abnormal liver function; the principle of hydration and nutritional support; the use of antibiotics, steroids and analgesics; the place of care; strategies employed in medical emergencies, and the withholding or withdrawal of life-sustaining procedures. Despite cultural differences, it seems widely agreed that appropriate hospice and palliative care be given within the framework of the principles of medical ethics. ${ }^{6}$

Major ethical principles in palliative care relate to:

1 Autonomy [respecting the values of the patient].

2 Beneficence and non-maleficence [applied to therapeutic strategy and decision making, such as truth-telling and choice of medication].

3 Justice, the balance between personal need and social resources, as in the selection of place of care. $^{89}$

In Taiwan lack of professional training and manpower may explain the lack of solution of some ethical dilemmas, inevitably affecting the quality of care of the terminally ill. ${ }^{10}$ This study investigated the most frequently encountered ethical dilemmas in the palliative care unit of the National Taiwan University Hospital, following the outcome of each dilemma and exploring factors related to it. The results suggest improvements which may promote the local quality of care.

\section{Patients and methods}

PATIENTS

Two hundred and forty-six consecutive patients, whose duration of stay in the hospice and palliative care unit of the National Taiwan University Hospital was longer than two days between July 1997 and the end of June 1998, were enrolled in the study. Health care workers recorded daily all ethical dilemmas in caring for each patient. Each dilemma 
was then examined in the weekly multidisciplinary team members' meeting.

\section{MEASUREMENTS}

The study was conducted in two parts.

\section{Field recording:}

For six months prior to the investigation all ethical dilemmas encountered during the care of patients were summarised in discussions at weekly multidisciplinary team meetings.

\section{Design and pretesting of a structured questionnaire:}

From the results of field recording, a questionnaire covering the most frequently encountered ethical dilemmas was designed. A group of experts [comprising physicians, a nursing supervisor, senior nurses, a psychologist and a social worker] were invited to review the questionnaire's comprehensiveness and applicability. The questionnaire was then piloted, and modified after weekly team discussion, for a month.

\section{Structured questionnaire}

A structured questionnaire was designed after analysis of the pilot and ethical dilemmas were cat-

Table 1 Demographic characteristics of patients

\begin{tabular}{|c|c|c|}
\hline Variables & $N$ & $\%$ \\
\hline \multicolumn{3}{|l|}{ Sex } \\
\hline Male & 128 & 52.0 \\
\hline Female & 118 & 48.0 \\
\hline \multicolumn{3}{|l|}{ Age } \\
\hline$\leqslant 18$ & 3 & 1.2 \\
\hline $19-35$ & 7 & 2.9 \\
\hline $36-64$ & 114 & 46.3 \\
\hline$\geqslant 65$ & 122 & 49.6 \\
\hline Mean \pm S.D. $60.6 \pm 14.7$ & & \\
\hline \multicolumn{3}{|l|}{ Primary tumour site } \\
\hline Lung & 51 & 20.7 \\
\hline Colon and rectum & 32 & 13.0 \\
\hline Liver & 27 & 11.0 \\
\hline Stomach & 22 & 8.9 \\
\hline Biliary - pancreas & 20 & 8.1 \\
\hline Head and neck & 16 & 6.5 \\
\hline Cervix & 12 & 4.9 \\
\hline Breast & 8 & 3.3 \\
\hline Prostate & 4 & 1.6 \\
\hline Brain & 3 & 1.2 \\
\hline Ovary & 2 & 0.8 \\
\hline Haematology & 1 & 0.4 \\
\hline Others & 48 & 19.5 \\
\hline \multicolumn{3}{|c|}{ Length of hospitalisation (days) } \\
\hline$\leqslant 7$ & 54 & 21.9 \\
\hline $8-30$ & 153 & 62.2 \\
\hline $31-60$ & 34 & 13.8 \\
\hline$\geqslant 61$ & 5 & 2.1 \\
\hline Mean \pm S.D. $19.3 \pm 16.0$ & & \\
\hline \multicolumn{3}{|l|}{ Outcome } \\
\hline Death & 161 & 65.4 \\
\hline Discharge & 85 & 34.6 \\
\hline \multicolumn{3}{|l|}{ No of admissions } \\
\hline 1 & 210 & 85.4 \\
\hline 2 & 30 & 12.2 \\
\hline \multirow[t]{2}{*}{3} & 6 & 2.4 \\
\hline & 246 & \\
\hline
\end{tabular}

egorised as follows: patient, family and medical team had different opinions on:

1) Telling the truth.

2) Place of care.

3) Therapeutic strategy [patient or the family could not accept the goal of treatment being directed towards "care" rather than "cure"].

4) Hydration and nutrition.

5) Blood transfusion.

6) Alternative treatment.

7) Terminal sedation.

8) Use of medication.

9) Other.

These dilemmas were graded by health care workers on a three-point scale as follows: 0, never happened; 1, happened, but was no longer a dilemma; 2, happened and was still a dilemma.

\section{Statistical analysis}

Data management and statistical analysis were performed using SPSS 6.0 statistical software. A frequency distribution was used to describe the demographic data and the distribution of each variable. Mean values and standard deviations were used to analyze the severity of each ethical dilemma. Data on ethical dilemmas were followed up on a weekly basis to assess dynamic change. Finally, $\chi$-square test and one way ANOVA were used to compare the differences between demographic variable and ethical dilemmas. A p value less than 0.05 was considered statistically significant.

\section{Results}

Table 1 shows that the number of males and females was similar (52\% and $48 \%$, respectively). Nearly half of the 246 patients were older than 65 years, and only three patients were younger than 18. The mean age was $60.6 \pm 14.7$ years. The primary sites of cancer were lung $(20.7 \%)$, colon $(13.0 \%)$ and liver $(11.0 \%)$. The mean length of hospital stay was $19.3 \pm 16$ days. A third of the patients $(34.6 \%)$ were discharged after symptom control and $36(14.6 \%)$ were admitted twice or more during the period.

Table 2 gives the type and frequency of ethical dilemmas encountered. Problems for the place of care occurred in $33 \%$ of patients, with truth-telling about the patients' terminal status in $32 \%$ of patients. Dilemmas concerning nutrition and therapeutic strategy occurred in about a quarter of patients; and with the use of medication in $19.1 \%$. Blood transfusion, terminal sedation and alternative treatment gave rise to fewer ethical dilemmas perhaps because of established therapeutic guidelines for these issues on the ward.

Although there was a dilemma to do with truthtelling for a quarter of patients during early hospitalisation, this gradually resolved with duration of stay (table 3). However, the longer the hospital stay, the greater the problem of place of care became. In 
Table 2 Types and frequency of ethical dilemmas during hospitalisation assessed by health care workers

\begin{tabular}{ll}
\hline Ethical dilemma & $N(\%)$ \\
\hline Place of care & $82(33.3)$ \\
Truth-telling & $79(32.1)$ \\
Hydration and nutrition & $62(25.2)$ \\
Therapeutic strategy & $61(24.8)$ \\
Use of medications & $47(19.1)$ \\
Blood transfusion & $18(7.3)$ \\
Terminal sedation & $18(7.3)$ \\
Alternative treatment & $11(4.5)$ \\
\hline
\end{tabular}

Total patients: 246

principle, in order to care for more patients, no patient may stay more than a month, unless there is medical indication. Problems in discharge from hospital occurred in over forty per cent of patients $(42.8 \%)$ in the fourth week of admission and another peak (50\%) occurred on the seventh week of admission.

Thirty-seven $(15.1 \%)$ patients insisted on cure as the goal of treatment on admission. However, by the fifth week in hospital all had changed their perspective regarding this goal. However, frustration with this issue appeared again in the following weeks, demonstrating that a fluctuation in acceptance of the goal of treatment creates increased need for clear communication between staff, patients and relatives.

It is more important to provide appropriate rather than excessive nutritional support in terminally ill patients. However, this general rule often conflicted with the traditional Chinese cultural traditions of medical care, which place heavy emphasis on the need for nutritional supplementation. In this study, $15 \%$ of patients had trouble with this issue during their early stay in hospital but these problems decreased with duration of stay, though among the eight patients who had been hospitalised for eight weeks, two still had problems with this issue.

Blood transfusion was given only if anaemia greatly reduced the quality of life of the patient, rather than in response to haemoglobin level. ${ }^{11}$ Few haemato-oncological patients were admitted to our ward, perhaps because of difficulty in justifying the indications for blood transfusion in the circumstances of our study. Dilemmas relating to blood transfusion occurred in only $2.8 \%$ of the patients in the first week of hospitalisation and the fluctuation in the frequency of these dilemmas during the study was negligible.

A 1996 Taiwanese study ${ }^{13}$ found that $81 \%$ of patients had used alternative treatments such as herbs $(51.4 \%)$, antineoplastic diets $(34.8 \%)$, faith healing $(18.1 \%)$, Qigong $(10.9 \%)$ and acupuncture $(5.1 \%)$ during the course of disease. In order to meet the expectations of patients and families, we accepted the patients' use of alternative treatment but only if it was inexpensive and non-invasive. Conflicts arising from the use of alternative treatment during the first week in hospital occurred in only $3.2 \%$ of the patients.

Trouble with the dilemma of terminal sedation during the first week in hospital occurred in $2.4 \%$ of patients. Terminal sedation was administered to those patients whose symptoms were refractory and this led to conflict (more about the timing of its use among patients, family and medical team, than its overall acceptability).

The most commonly observed problems with medication were: using herb treatment instead of prescribed medicine; misunderstandings about the purpose of morphine, and worry about its untoward effects. During the first week in hospital more than one tenth $(12.2 \%)$ of patients had problems with the use of medication and the incidence of this problem fluctuated later.

We also compared the demographic characteristics of patients with the incidence of ethical dilemmas. There was no apparent association of gender with change in any of the ethical dilemmas. However, the problems with truth-telling occurred in $42.6 \%$ of patients older than 65 years $(p<0.05)$. The conflict of transfusion occurred in all patients younger than 18 years. Patients in the age range of 19 to 35 years had more problems than other age groups with the issue of terminal sedation $(p<0.05)$. In half the patients with biliarypancreatic cancer and one third of the patients with lung, liver or cervical cancer there were problems relating to telling the truth. More than forty per cent $(43.8 \%)$ of the patients with head and neck cancers had problems accepting or complying with recommended discharge plans; this may be related to the need for special care for these patients, such as wound dressing or management of bleeding. There was no apparent difference among primary sites of cancer and the incidence of ethical dilemmas related to therapeutic strategy. Nearly

Table 3 Change in the frequency of ethical dilemmas during the course of hospitalisation (\%)

\begin{tabular}{|c|c|c|c|c|c|c|c|c|c|c|c|c|}
\hline Ethical dilemma & $\begin{array}{l}\text { Week 1 } \\
(N=246)\end{array}$ & $\begin{array}{l}\text { Week } 2 \\
(N=156)\end{array}$ & $\begin{array}{l}\text { Week } 3 \\
(N=102)\end{array}$ & $\begin{array}{l}\text { Week } 4 \\
(N=63)\end{array}$ & $\begin{array}{l}\text { Week } 5 \\
(N=31)\end{array}$ & $\begin{array}{l}\text { Week } 6 \\
(N=18)\end{array}$ & $\begin{array}{l}\text { Week } 7 \\
(N=14)\end{array}$ & $\begin{array}{l}\text { Week } 8 \\
(N=8)\end{array}$ & $\begin{array}{l}\text { Week } 9 \\
(N=6)\end{array}$ & $\begin{array}{l}\text { Week } 10 \\
(N=5)\end{array}$ & $\begin{array}{l}\text { Week } 11 \\
(N=4)\end{array}$ & $\begin{array}{l}\text { Week } 12 \\
(N=1)\end{array}$ \\
\hline Truth-telling & 25.6 & 17.9 & 20.5 & 15.8 & 6.4 & 5.6 & 7.1 & 0.0 & 0.0 & 0.0 & 0.0 & 0.0 \\
\hline Place of care & 9.0 & 21.8 & 30.4 & 42.8 & 22.6 & 30.0 & 50.0 & 37.5 & 16.7 & 20.0 & 0.0 & 0.0 \\
\hline Therapeutic strategy & 15.1 & 14.7 & 13.8 & 11.1 & 0.0 & 11.2 & 7.1 & 12.5 & 0.0 & 0.0 & 0.0 & 0.0 \\
\hline Hydration and nutrition & 15.0 & 13.4 & 6.8 & 11.1 & 9.7 & 5.6 & 7.1 & 25.0 & 0.0 & 0.0 & 0.0 & 0.0 \\
\hline Blood transfusion & 2.8 & 3.2 & 4.9 & 3.2 & 0.0 & 0.0 & 7.1 & 12.5 & 0.0 & 20.0 & 0.0 & 0.0 \\
\hline Alternative treatment & 3.2 & 1.3 & 2.0 & 4.8 & 0.0 & 0.0 & 0.0 & 0.0 & 0.0 & 0.0 & 0.0 & 0.0 \\
\hline Terminal sedation & 2.4 & 5.1 & 3.9 & 3.2 & 0.0 & 0.0 & 0.0 & 12.5 & 0.0 & 0.0 & 0.0 & 0.0 \\
\hline Use of medications & 12.2 & 9.6 & 10.8 & 6.4 & 16.1 & 0.0 & 7.1 & 12.5 & 0.0 & 0.0 & 25.0 & 0.0 \\
\hline
\end{tabular}

Total patients: 246 
Table 4 The frequency of ethical dilemmas noted in the last week of hospitalisation

\begin{tabular}{llll}
\hline & $\begin{array}{l}\text { Never } \\
\text { happened } \\
\text { N(\%) }\end{array}$ & $\begin{array}{l}\text { Noted but not } \\
\text { bothersome } \\
\text { again N }(\%)\end{array}$ & $\begin{array}{l}\text { Noted and } \\
\text { still } \\
\text { bothersome } \\
\text { N(\%) }\end{array}$ \\
Ethical dilemma & $204(82.9)$ & $16(6.5)$ & $26(10.6)$ \\
\hline Truth-telling & $189(76.8)$ & $30(12.2)$ & $27(11.0)$ \\
Place of care & $218(88.6)$ & $17(6.9)$ & $11(4.5)$ \\
Therapeutic strategy & $216(87.8)$ & $22(8.9)$ & $8(3.3)$ \\
Hydration and nutrition & $217(96.3)$ & $8(3.3)$ & $1(0.4)$ \\
Blood transfusion & $237(239(97.2)$ & $5(2.0)$ & $2(0.8)$ \\
Alternative treatment & $238(96.8)$ & $4(1.6)$ & $4(1.6)$ \\
Terminal sedation & $238(5)$ & & $12(4.9)$ \\
Use of medications & $219(89.0)$ & $15(6.1)$ &
\end{tabular}

Total patients: 246

sixty per cent $(59.1 \%)$ of patients with gastric cancer had ethical problems associated with hydration and nutrition, as did about a third of patients with either head and neck $(37.5 \%)$ or colon cancer $(34.4 \%)$. This finding may be related to common problems of dysphagia or bowel obstruction.

Regarding the length of stay, nearly two-thirds $(64.1 \%)$ of patients in hospital from 31 to 60 days had trouble in accepting or complying with recommended discharge plans, and sixty per cent of those who stayed more than 61 days also had trouble with this issue. As for blood transfusion and terminal sedation, patients staying in for more than two months had a higher $(40 \%)$ incidence of conflict in relation to these issues. Of patients who stayed on our ward for less than three days in 31.6 per cent there was a dilemma concerning therapeutic strategy.

The mean number of ethical dilemmas encountered for each patient was 1.5. More were reported among female patients than males (1.70 v 1.41) Patients younger than 18 years had more ethical dilemmas than the other age groups $(2.33 v 1.53)$. Patients who received inpatient care for longer than two months had more ethical dilemmas $(3.00 \pm 1.73, \mathrm{p}<0.001)$ than others. Patients with gastric cancer or head/neck cancer had more ethical dilemmas $(1.96 \pm 1.62,1.75 \pm 1.73)$ than patients with other primary sites of cancer.

Problems with discharge during the last week of hospitalisation occurred in one fourth of patients $(23.2 \%)$ (table 4$)$. In addition, trouble with truth-telling occurred with $17.1 \%$ of patients, and in $10.6 \%$ of cases a solution to this problem was never reached. It was somewhat surprising that $11.4 \%$ of the patients or their families still requested curative treatment rather than palliative care in the last week of hospitalisation.

\section{Discussion}

In this study, one-third (34.6\%) of the terminally ill patients were discharged after control of symptoms. This contradicts the common perception that a Taiwanese hospice serves as a place only to wait for death. Our medical staff not only relieved the physical and psychological distress of the patients (beneficence), but also helped the patients to return home once their conditions became stable, accord- ing to their expectations (autonomy). However, ethical dilemmas surrounding the discharge plan occurred in one third of the patients in our study. In oriental culture, it is common practice not to disclose the truth of the illness especially to a terminal cancer patient, on the basis of nonmaleficence. This mutual pretence prevails because both sides are unwilling to hurt each other and lack knowledge of how to communicate with each other. Nevertheless, evidence suggests that a good death is easier to obtain if patients have enough time to arrange their affairs. Our previous study found that a "good death score" was higher if the patient was aware of his or her own imminent death. ${ }^{14}$ However, we found that the intention and process of telling the truth were often interrupted by relatives, although this could be gradually resolved through continuing communication. In this study, fewer than ten per cent of patients in their seventh week in hospital had this dilemma compared to one quarter of patients in the first week, and it was never an issue after the eighth week. Another commonly encountered ethical dilemma was the demand for hydration and nutrition. Because patients with terminal cancer often develop anorexia or dysphagia, parenteral fluids and nutrition are usually requested by the families of these patients. However, inappropriate hydration and nutrition may further increase patients' distress by aggravating conditions such as ascites, limb oedema and gastrointestinal secretions..$^{15}$ Moreover, parenteral nutrition can also be absorbed by cancer cells, ${ }^{17}$ violating the principle of non-maleficence. Meanwhile, use of parenteral hydration and nutrition too early may replace the family's delicate care and make the relationship between the patient and the family, and caregivers, more aloof. Improving the quality of life by encouraging more interaction between patient and family is an essential part of palliative care. One fourth of patients experienced difficulty in deciding on the appropriate goal of treatment between "cure" and "pursuing better quality of life" and this was not resolved by a longer period of hospitalisation. Emphasis on the need for communication appeared to be the only useful strategy in resolving this issue. Perhaps because of the widespread misunderstanding of the medical role of opiumderived compounds since the opium wars in China in 1840, some patients and families still preferred to tolerate pain rather than use morphine. Poor pain control resulted in an unnecessary deterioration of physical function and life quality, which unfortunately was common in this patient group. Another important factor affecting pain control was the use of herbal drugs, in accordance with Taiwanese beliefs, which may have adverse interactions with Western medication.

The study found that a mean of 1.5 important ethical dilemmas were encountered by each patient during his or her stay. These dilemmas not only puzzled the medical team, but also impeded the pursuit of better quality of life and a meaningful and peaceful death. Further investigations are 
needed to establish guidelines suitable for dealing with these dilemmas.

In conclusion, many ethical dilemmas were encountered in caring for the terminally ill which troubled the patient, the family and the medical staff. Good communication between the medical staff and patients and family is essential if attitudes towards these ethical dilemmas are to be improved. Professional development based on good research evidence may help to solve these dilemmas. Education which focuses on medical ethics is crucial for appropriate decision making in palliative care. It is also important to emphasise that relieving the suffering of the terminally ill and providing them with adequate support, is an essential part of the health care system.

\section{Acknowledgement}

This research was supported by a grant (DOH87TD-1079) from the Department of Health, the Executive Yuan, Taiwan. We are indebted to the faculties of the Department of Family Medicine, National Taiwan University Hospital, and also to Ms Y C Chang, Ms C T Wang and Ms C H Teng for their secretarial support.

Tai-Yuan Chiu, MD, is Assistant Professor in the Department of Social Medicine, College of Medicine, National Taiwan University and Medical Director of the Hospice and Palliative Care Unit, in the Department of Family Medicine, National Taiwan University Hospital, Taipei, Taiwan. Wen-Yu Hu, MSN, is Nursing Supervisor of the Hospice and Palliative Care Unit at National Taiwan University Hospital, and Assistant Professor in the Department of Nursing Science, College of Medicine, National Taiwan University. Shao-Yi Cheng, MD, is Consultant Physician at the Hospice and Palliative Care Unit at National Taiwan University Hospital. Ching-Yu Chen, MD, is Consultant Physician at the Hospice and Palliative Care Unit at National
Taiwan University Hospital and the Professor and Chief of Department of Family Medicine,College of Medicine, National Taiwan University.

\section{References}

1 Wanzer SH, Federman DD, Adelstein SJ, Cassem EH, Cranford RE, Hook EW, et al. The physician's responsibility toward hopelessly ill patients: a second look. New England fournal of Medicine 1989;320:844-9.

2 Quill TE, Lo B, Brock DW. Palliative options of last resort: a comparison of voluntarily stopping eating and drinking, terminal sedation, physician-assisted suicide, and voluntary active euthanasia. The fournal of the American Medical Association 1997;278:2099-104.

3 Department of Health, The Executive Yuan, Republic of China. Vital statistics, Republic of China 1996. Taipei: Department of Health, The Executive Yuan, Republic of China, 1997.

4 Bradshaw A. The spiritual dimension of hospice: the seculariBradshaw A. The spiritual dimension of hospice: the seculari-
zation of an ideal [review]. Social Science and Medicine 1996;43: 409-19.

5 Kinzbrunner BM. Ethical dilemmas in hospice and palliative care. Supportive Care in Cancer 1995;3:28-36.

6 Finlay I. Difficult decisions in palliative care. British fournal of Hospital Medicine 1996;56:264-7.

7 Henkelman WJ, Dallinis PM. A protocol for palliative care measures. Nursing Management 1998;29:36C-G.

8 Randall F, Downie RS. Palliative care ethics: a good companion. New York: Oxford University Press, 1996.

9 Beauchamp TL, Childress JF. Principles of biomedical ethics [4th ed]. New York: Oxford University Press, 1994

10 Chiu TY, Hu WY, Tsay FC, Chou LL, Yao CA, Chen CY. Ethical dilemmas in palliative care: a multicenter study. Formosan fournal of Medicine 1998;2:633-40.

11 Monti M, Castellani L, Berlusconi A, Cunietti E. Use of red blood cell transfusions in terminally ill cancer patients admitted to a palliative care unit. fournal of Pain and Symptom to a palliative care unit.

12 Gleeson C, Spencer D. Blood transfusion and its benefit in palliative care. Palliative Medicine 1995;9:307-13.

13 Hsin LS, Chiu TY, Hu WY, Cheng SY, Cheng CY. A study of alternative treatment in terminal cancer patients. Chinese fournal of Family Medicine 1996;6:127-37.

14 Cheng SY, Chiu TY, Hu WY, Guo FR, Wang Y, Chou LL, et al. A pilot study of good death in terminal cancer patients. Chinese fournal of Family Medicine 1996;6:83-92.

15 McQuillan R, Finlay I. Dehydration in dying patients. Palliative Medicine 1995;9:341.

16 Fainsinger RL, Bruera E. When to treat dehydration in a terminally ill patient? Supportive Care in Cancer 1997;5:205-11.

17 Torosian M, Daly J. Nutritional support in the cancer bearing host. Cancer 1986;58:1915-29. 